\title{
Calcific Tendinopathy of the Shoulder
}

\author{
Nathalie J. Bureau, MD, FRCPC 1 \\ ${ }^{1}$ Department of Radiology and Research Center, Centre Hospitalier de \\ I'Université de Montréal (CHUM), Montreal, Quebec, Canada \\ Semin Musculoskelet Radiol 2013;17:80-84.
}

\begin{abstract}
Address for correspondence Nathalie J. Bureau, MD, Department of Radiology and Research Center, Centre Hospitalier de l'Université de Montréal (CHUM), 1058 Saint-Denis St., Montreal, Quebec, H2X 3J4 Canada (e-mail: nathalie.bureau@umontreal.ca).
\end{abstract}
Abstract Keywords
- calcific tendinopathy
- shoulder
- ultrasound
- lavage

This review article presents the current knowledge on the epidemiology and the pathogenesis of calcific tendinopathy of the shoulder and discusses the clinical presentation in relation to the stage of the disease process and the appearance of the calcific deposits. The outcome and the available treatment modalities for this common shoulder disorder are also examined, emphasizing the technique of percutaneous lavage and aspiration under ultrasound guidance.
Calcific tendinopathy involving the substance of the tendon is particularly common in the rotator cuff, whereas insertional calcific tendinopathy involving the deposition of calcium at the enthesis affects predominantly the Achilles tendon, the common extensor tendon at the elbow, the gluteus medius and minimus at the greater trochanter, and the patellar tendon. Calcific tendinopathy and insertional calcific tendinopathy appear to be caused by two distinct pathogenetic mechanisms. ${ }^{1}$

\section{Epidemiology, Pathogenesis, and Clinical Presentation}

Calcific tendinopathy of the rotator cuff is a common disorder that affects predominantly the 40- to 60-year-old age group. Women appear to be more commonly affected than men, and contrary to popular belief, individuals involved in strenuous manual labor or athletic activities are not more commonly affected than those leading a sedentary life. ${ }^{2}$ There appears to be a slight preponderance in the dominant shoulder, but the nondominant, and even both shoulders, may be affected.

Rotator cuff calcific tendinopathy is caused by the deposition of hydroxyapatite crystals, ${ }^{3}$ most commonly in the supraspinatus and infraspinatus tendons. The subscapularis and teres minor tendons may also be involved, but these calcific deposits tend to be less frequently symptomatic unless they are very large.

The pathogenesis of calcific tendinopathy is still a matter of controversy. According to Uhthoff and colleagues, the deposition of calcium apatite crystals within the substance of the tendon is not a degenerative process but one that is actively mediated by cells in a viable tendon. ${ }^{4}$ The theory proposed by Uhthoff et al considers the clinical course, the morphological aspects, and the histologic features of the disease. Uhthoff and colleagues described three distinct stages in the disease process, namely the precalcific, calcific, and postcalcific stages. Depending on the stage of the disease, the imaging appearance and physical consistency of the calcification differ significantly as do patient symptoms. ${ }^{5}$

According to this theory, during the precalcific stage, fibrocartilaginous metaplasia of tenocytes with the production of proteoglycan occurs in generally avascular areas. This concept appears to be supported by histologic examinations. ${ }^{6}$ Conversely, the stimuli that cause the initiation of the precalcific stage remain unknown.

The following calcific stage consists of three phases: the formative, resting, and resorptive phases. During the formative phase, calcium crystals are deposited into matrix vesicles that coalesce and gradually form foci of calcification. During the resting phase, the deposition of calcium is terminated and fibrocollagenous tissue encircles and isolates the foci of calcification. As demonstrated at surgery, during these two phases, the calcium deposits have a chalk-like appearance. ${ }^{4}$ The formative and resting phases are chronic, usually lasting for a few years, and they may be associated with intermittent or constant varying degrees of pain at rest or with movement, especially abduction. Many individuals with radiographic evidence of calcific tendinopathy, however, are asymptomatic. ${ }^{2}$ In our experience, the larger calcifications and those located at the confluence of the supraspinatus and infraspinatus tendons tend to be most symptomatic. The symptomatic calcific deposits may cause focal thickening of the tendon (-Fig. 1), they may
Copyright @ 2013 by Thieme Medical Publishers, Inc., 333 Seventh Avenue, New York, NY 10001, USA. Tel: +1(212) 584-4662.
DOI http://dx.doi.org/ 10.1055/s-0033-1333941. ISSN 1089-7860. 


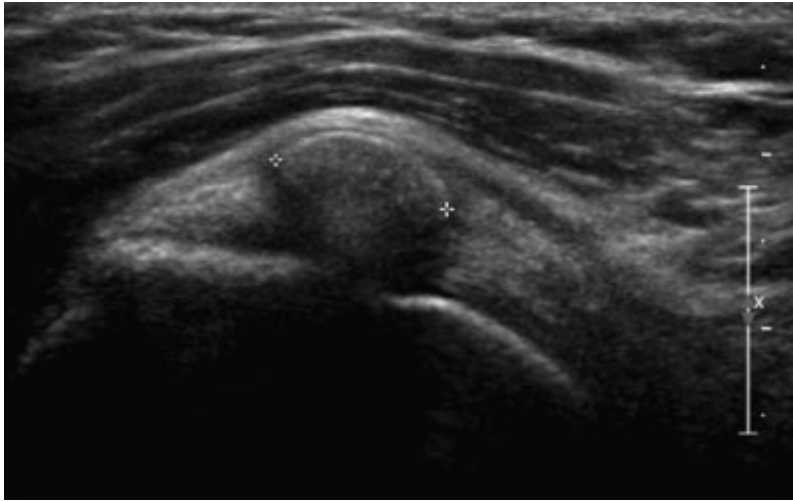

Fig. 1 Sagittal ultrasound scan of the infraspinatus tendon shows an intratendinous calcific deposit (between cursors) causing focal thickening and a convexity of the superficial surface of the tendon.

elicit pain when probed percutaneously, and they may mechanically contribute to a subacromial (supraspinatus, infraspinatus) or a subcoracoid (subscapularis) impingement syndrome. When associated with an impingement syndrome, a concomitant subacromial bursopathy is often present. Some authors have showed that the presence of a power Doppler signal near the tendon calcification is more common in patients with symptomatic calcifications than in individuals with asymptomatic calcifications. ${ }^{7}$ During the resting phase of the disease, the calcifications tend to be well circumscribed and discrete when examined radiographically (-Fig. 2 ), and they often produce significant acoustic shadowing at ultrasound (US) $)^{8}$ (-Fig. 3 ).

The resorptive phase is the last of the three phases in the calcific stage, during which spontaneous resorption of calcium occurs with the formation of thin-walled vascular channels that surround the calcific deposits, associated with a reaction driven by macrophages and giant cells that phagocytose and remove the calcium. The factors that trigger the resorptive phase also remain unknown. It has been demonstrated at surgery that, during this phase, the calcific deposits have a toothpaste-like appearance. ${ }^{4}$ During this phase,

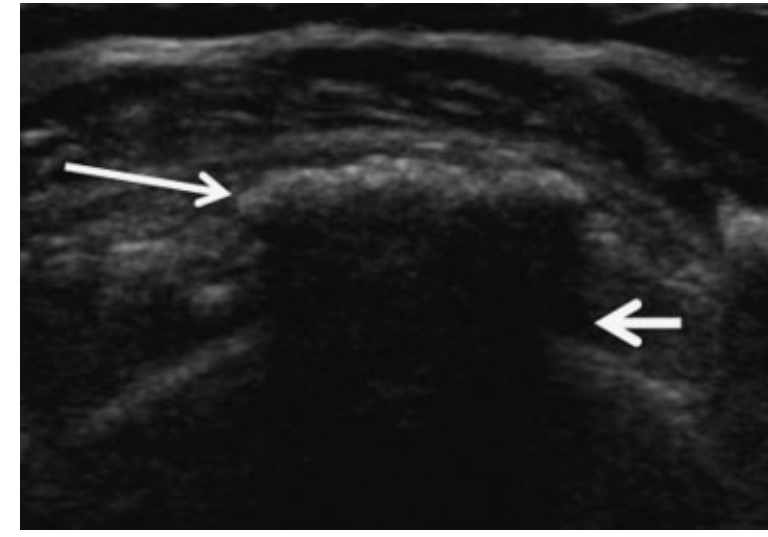

Fig. 3 Sagittal ultrasound scan of the posterosuperior cuff demonstrates a large hyperechoic calcific deposit (long arrow) located at the confluence of the supraspinatus and infraspinatus tendons, causing significant acoustic shadowing on the humeral cortex (short arrow).

patients may present clinically with an acute inflammatory shoulder syndrome characterized by severe pain and very limited range of motion caused by shedding of calcium crystals into the adjacent subacromial bursa ( - Fig. 4), within the rotator cuff tendons or even into the adjacent bone (-Fig. 5). This hyperalgic syndrome typically lasts for 2 weeks and is usually followed by substantial clinical improvement. During this phase, the calcifications appear ill defined on radiographs and produce little or no acoustic shadowing at US (-Fig. 6). When percutaneously aspirated, these calcific deposits typically are soft with a slurry-like consistency. ${ }^{9}$ Finally, during the postcalcific stage, reparation of the tendon occurs with remodeling by fibroblasts and new vascular channels that produce collagen and mature scar tissue.

\section{Outcome and Treatment}

In most cases, calcific tendinopathy is a self-limiting condition, in which the calcification spontaneously resorbs after a period of a few years or is treated successfully conservatively. ${ }^{10}$ In the formative and resting phases of the disease, if conservative measures fail and the patient presents with progressive
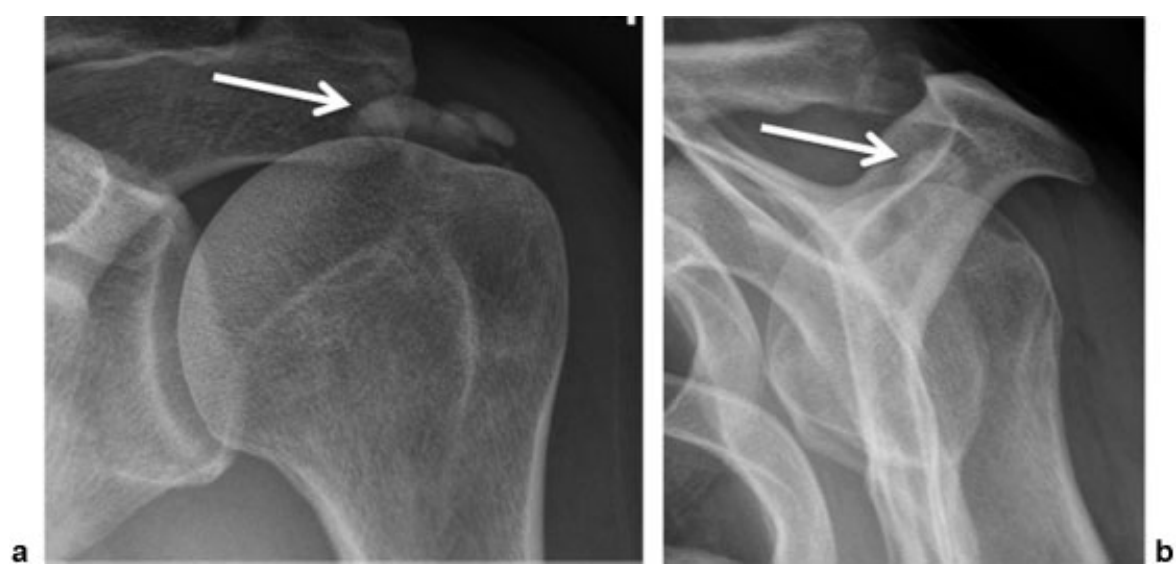

Fig. 2 (a) Anteroposterior and (b) lateral radiographs of the left shoulder demonstrate a large multilobular calcific deposit (arrow) in the infraspinatus tendon. 


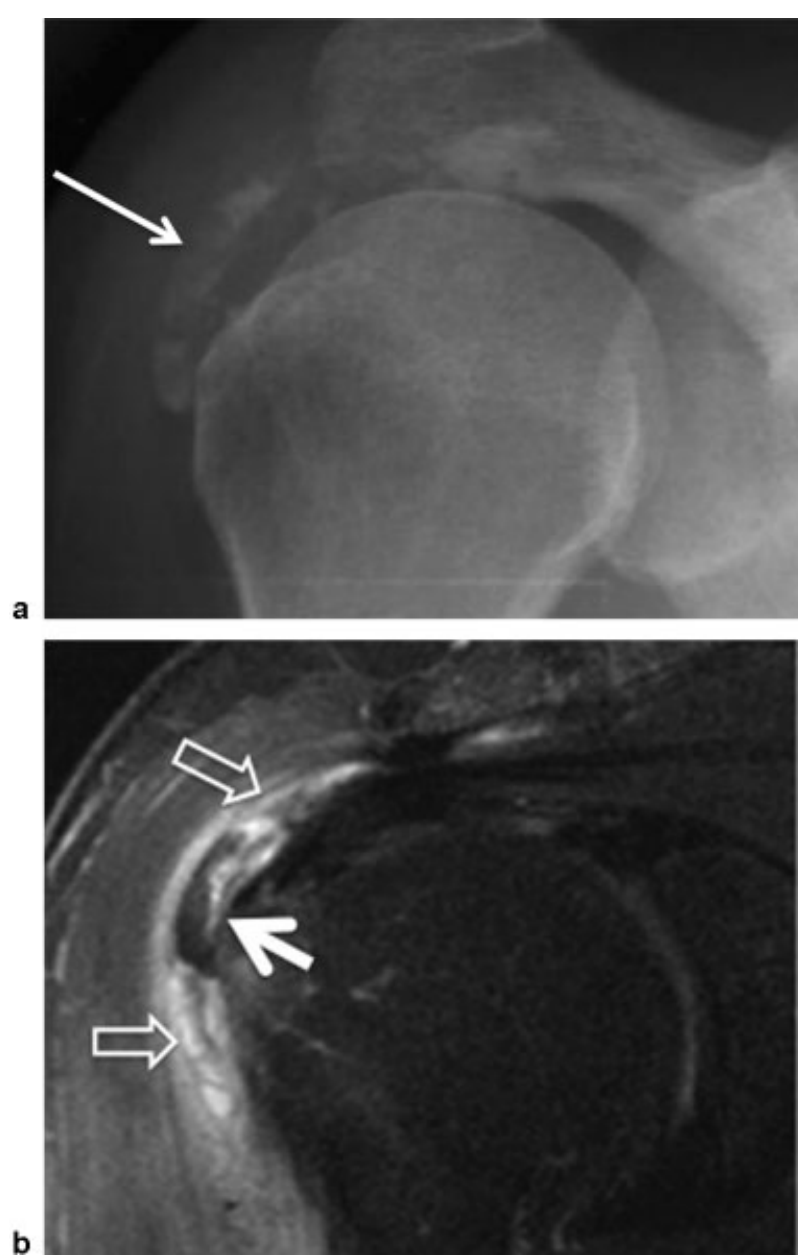

Fig. 4 (a) Anteroposterior radiograph of the right shoulder shows ongoing resorption of a large calcification into the subacromial-subdeltoid bursa (long arrow). (b) Corresponding coronal oblique

T2-weighted with fat suppression MR image depicts the hypointense calcification (short arrow) in the hyperintense subacromial-subdeltoid bursitis (open arrows).

symptoms interfering with daily living activities, removal of calcific deposits may be indicated. ${ }^{4}$ The resolution of calcification has been shown to correlate well with clinical improvement of symptoms, and therefore various treatments have been devised to promote their removal. Arthroscopic removal of calcific deposits has shown successful results with significant improvement in shoulder function at 24 months and progressive decrease in pain level up to a year after the procedure. ${ }^{11}$ There is evidence supporting the use of extracorporeal shock-wave therapy (ESWT) in calcific tendinopathy of the rotator cuff. For instance, a randomized placebo-controlled study, in which 134 patients (of 144 enrolled) completed the treatment and the 6-month follow-up, suggested a beneficial effect of high-energy ESWT over 6 months for shoulder function, self-rated pain, and diminished size of calcifications, compared with the sham treatment. ${ }^{12}$ Another randomized double-blind sham-controlled trial compared the effects of acetic acid iontophoresis (AAI) and physiotherapy to physiotherapy alone in 27 patients (of 36 enrolled) with calcific tendinopathy. ${ }^{13}$ This study concluded that treatment of calcific tendinopathy of the rotator cuff with AAI and physiotherapy
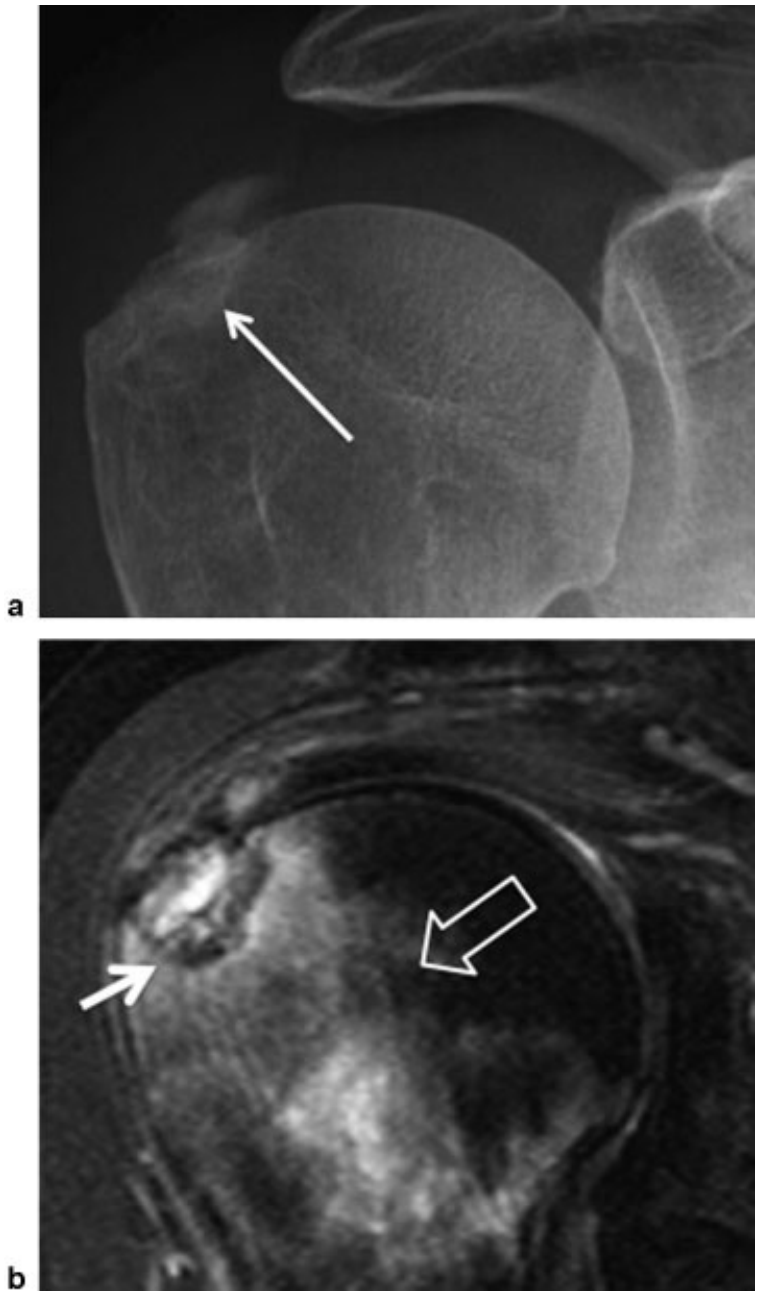

Fig. 5 (a) Anteroposterior radiograph of the right shoulder demonstrates a calcific deposit in the resorptive phase that has eroded the humeral cortex and is migrating into the bone (long arrow).

(b) Corresponding coronal oblique T2-weighted MR image with fat suppression shows the cortical erosion (short arrow) and the hyperintense reactive bone marrow edema (open arrow).

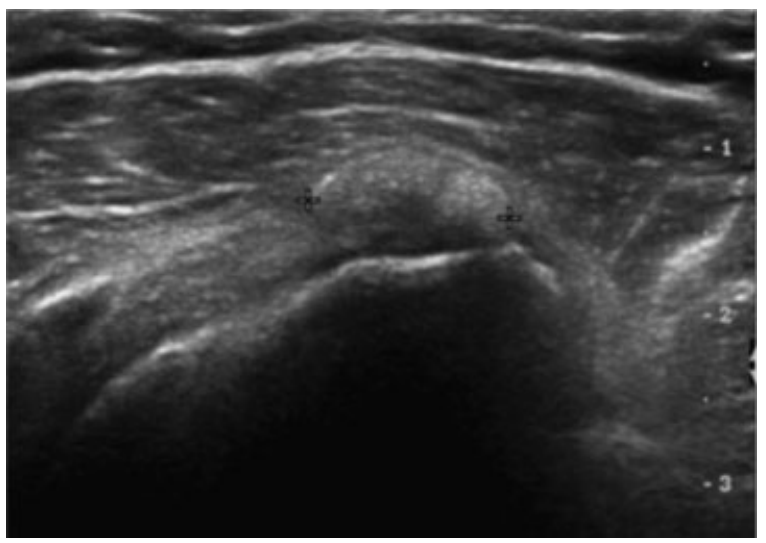

Fig. 6 Longitudinal ultrasound scan of the left subscapularis tendon showing an almost isoechoic calcification (between cursors). Note the absence of posterior acoustic shadowing. This appearance suggests that the calcification has entered the resorptive phase. 
did not result in better clinical or radiographic effects than those observed in patients treated with physiotherapy alone.

Fluoroscopy-guided needle lavage and aspiration of rotator cuff calcifications was first described $>30$ years ago. ${ }^{14}$ In 1995 , Farin and colleagues described a two-needle US-guided technique to treat calcific tendinopathy. ${ }^{15}$ One year later, the same group presented their results in 61 shoulders with calcific tendinopathy treated with this US-guided technique. ${ }^{9}$ Their clinical results at 1-year follow-up were excellent in $74 \%$, moderate in $16 \%$, and poor in $10 \%$ of patients, respectively. More recent studies using either a one-needle ${ }^{16,17}$ or a twoneedle ${ }^{18}$ US-guided technique have reported that most patients experienced significant or complete improvement in pain and disability. More recently, a study by Serafini and colleagues suggested that treated patients had better outcomes than did untreated patients at 1 year. ${ }^{18}$ In a prospective study, Sconfienza and colleagues compared the use of room-temperature saline to warm saline $\left(42^{\circ} \mathrm{C}\right)$ in the US-guided lavage and aspiration treatment of rotator cuff calcific tendinopathy in 462 patients. ${ }^{19}$ Their study suggests that the use of warm saline reduces procedure time by improving calcium dissolution. They also found a lower frequency of postprocedural subacromial-subdeltoid bursitis in the warm saline group as compared with the group treated with room-temperature saline.

\section{Ultrasound-Guided Lavage and Aspiration Technique}

US-guided lavage and aspiration techniques vary greatly, and there is no accepted standardized methodology. At our

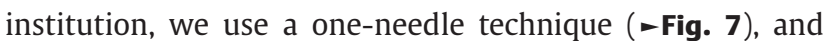
we routinely perform a complete shoulder US examination before any intervention. ${ }^{16}$ This is done to ensure that there are no coexisting disorders such as rotator cuff tears or adhesive capsulitis, and to confirm that the calcific tendinopathy is the cause of the patient's symptoms. For the intervention, the patient is placed into an oblique lateral decubitus position opposite the affected side. Depending on the location of the calcification in the rotator cuff, the arm is positioned for adequate visualization of the calcification (neutral or internal rotation for the supraspinatus and infraspinatus tendons, external rotation for the subscapularis tendon).

After sterile preparation of the skin and equipment, the calcification is targeted under US from a sagittal oblique posteroanterior approach using a 22-gauge needle connected to a $10-\mathrm{mL}$ syringe filled with $1 \%$ lidocaine. As the needle is advanced, the skin, the subcutaneous tissues, and the subacromial bursa are injected with lidocaine for local anesthesia. Approximately 4 to $6 \mathrm{~mL}$ of lidocaine are used. Then the 22-gauge needle is retrieved and replaced by an 18-gauge needle, which, in turn, is connected to the $10-\mathrm{mL}$ syringe filled with $1 \%$ lidocaine, and using the same approach, under US monitoring, the larger bore needle is advanced to penetrate into the calcification. Importantly, the calcification should be punctured only once, and lidocaine is injected into the calcification without first aspirating, to prevent the needle tip from becoming obstructed. Starting the lavage, the first injection is usually forceful and creates a cleavage in the substance of the calcification. The lavage is performed

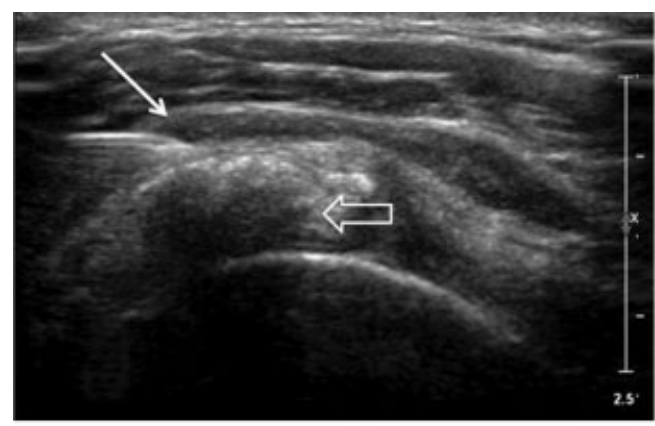

a

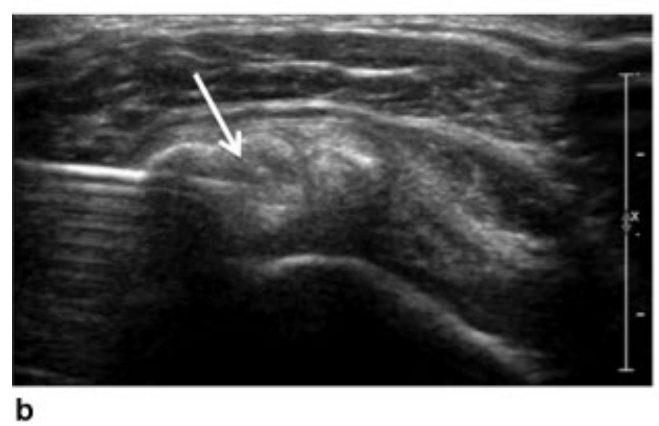

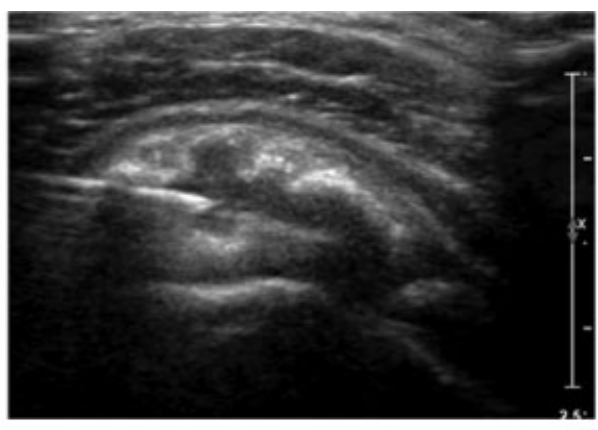

C
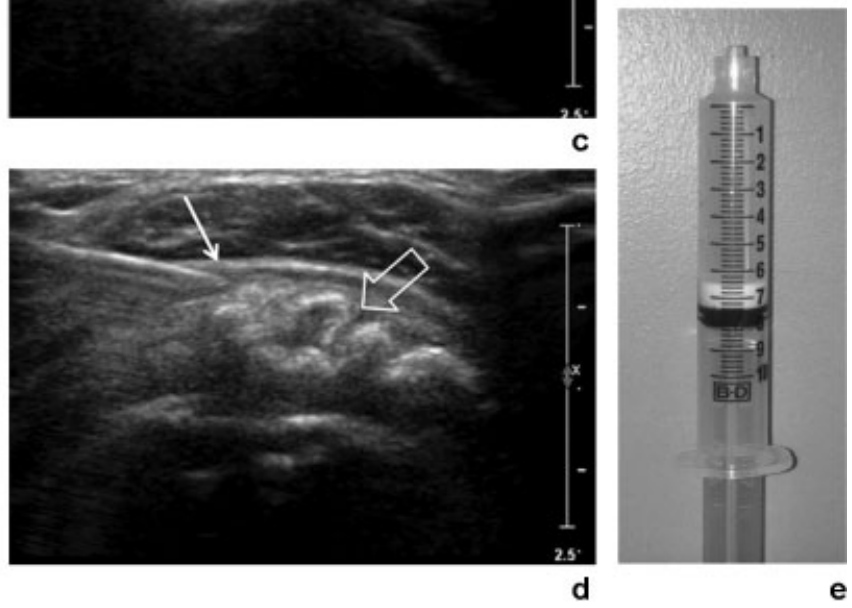

Fig. 7 (a) Sagittal ultrasound scan of the posterosuperior cuff showing injection of lidocaine $1 \%$ into the subacromial bursa (arrow) using a 22-gauge needle, at the level of a large rotator cuff calcification (open arrow). (b) Then, an 18-gauge needle connected to a 10 -mL syringe filled with lidocaine $1 \%$ is advanced into the calcification (arrow). Note the near-horizontal orientation of the needle. (c) Progressive lavage and aspiration of the calcium has created a cavity inside the calcification, leaving an irregular hyperechoic calcific rim. (d) After completion of the lavage, the 18-gauge needle is partly withdrawn and positioned into the subacromial bursa for the injection of a mixture of cortisone and bupivacaine 5\% (arrow). Note the residual decompressed, fragmented hyperechoic calcific deposit (open arrow). (e) At the end of the procedure, the syringe showing the amount of calcium that was retrieved. 
with several short injections, each followed by release of pressure on the plunger to let lidocaine and calcium fragments evacuate back into the syringe. The syringe can be held horizontal or below horizontal to facilitate return of the aspirated calcific material. Also, new lidocaine-filled syringes can be exchanged for the old one when it is filled with calcium crystals. Lavage and aspiration should be continued until no more calcium can be aspirated. It may not be possible to aspirate very hard calcifications, in which case the calcification can be fragmented by injecting it and probing it gently by moving the needle tip and rotating the syringe. At the end of the procedure, when no more crystals are coming into the syringe, not all the calcifications will be gone. The needle is then withdrawn into the subacromial bursa where a combination of $20 \mathrm{mg} / \mathrm{mL}$ of triamcinolone and $2.5 \mathrm{~mL}$ of bupivacaine $0.5 \%$ is injected to mitigate the risk of postprocedural bursitis. The entire procedure usually lasts approximately 20 minutes. The procedure is usually not painful, although the patient may feel some discomfort from the pumping effect of the lavage. Rarely, some patients may experience a vagal reaction.

After the procedure, the patient is instructed to apply ice on the shoulder, intermittently according to the usual protocol, as needed, and take analgesics as needed, although most patients do not experience any significant postprocedural pain. Patients are also instructed to limit strenuous exercises involving the shoulder for up to 4 weeks. A follow-up appointment is scheduled for 8 weeks after the treatment and includes assessment of clinical improvement and repeat US. If the patient presents with residual pain and restricted range of motion, which is sometimes the case with larger calcifications, a second percutaneous treatment is usually performed. Other patients may also present with recurring pain after a period of complete resolution of symptoms. This is usually caused by the dispersing calcium crystals inciting a subacromial bursitis. In those cases, we perform a subacromial steroid injection.

\section{Conclusion}

Understanding the evolution of calcific tendinopathy, which correlates with the clinical symptoms and the imaging features of the calcifications, helps to tailor management and to determine prognosis of this disorder. Percutaneous calcium aspiration is a minimally invasive and effective treatment of calcific tendinopathy. Rarely, if conservative treatment fails, surgery may become necessary.

\section{References}

1 Oliva F, Via AG, Maffulli N. Physiopathology of intratendinous calcific deposition. BMC Med 2012;10(1):95
2 Speed CA, Hazleman BL. Calcific tendinitis of the shoulder. N Engl J Med 1999;340(20):1582-1584

3 Gärtner J, Simons B. Analysis of calcific deposits in calcifying tendinitis. Clin Orthop Relat Res 1990;(254):111-120

4 Uhthoff HK, Loehr JW. Calcific tendinopathy of the rotator cuff: pathogenesis, diagnosis, and management. J Am Acad Orthop Surg 1997;5(4):183-191

5 Chiou HJ, Hung SC, Lin SY, Wei YS, Li MJ. Correlations among mineral components, progressive calcification process and clinical symptoms of calcific tendonitis. Rheumatology (Oxford) 2010;49 (3):548-555

6 Archer RS, Bayley JI, Archer CW, Ali SY. Cell and matrix changes associated with pathological calcification of the human rotator cuff tendons. J Anat 1993;182(Pt 1):1-11

7 Le Goff B, Berthelot JM, Guillot P, Glémarec J, Maugars Y. Assessment of calcific tendonitis of rotator cuff by ultrasonography: comparison between symptomatic and asymptomatic shoulders. Joint Bone Spine 2010;77(3):258-263

8 Farin PU, Jaroma H. Sonographic findings of rotator cuff calcifications. J Ultrasound Med 1995;14(1):7-14

9 Farin PU, Räsänen H, Jaroma H, Harju A. Rotator cuff calcifications: treatment with ultrasound-guided percutaneous needle aspiration and lavage. Skeletal Radiol 1996;25(6):551-554

10 Cho NS, Lee BG, Rhee YG. Radiologic course of the calcific deposits in calcific tendinitis of the shoulder: does the initial radiologic aspect affect the final results? J Shoulder Elbow Surg 2010;19(2): 267-272

11 Seil R, Litzenburger H, Kohn D, Rupp S. Arthroscopic treatment of chronically painful calcifying tendinitis of the supraspinatus tendon. Arthroscopy 2006;22(5):521-527

12 Gerdesmeyer L, Wagenpfeil S, Haake M, et al. Extracorporeal shock wave therapy for the treatment of chronic calcifying tendonitis of the rotator cuff: a randomized controlled trial. JAMA 2003;290 (19):2573-2580

13 Leduc BE, Caya J, Tremblay S, Bureau NJ, Dumont M. Treatment of calcifying tendinitis of the shoulder by acetic acid iontophoresis: a double-blind randomized controlled trial. Arch Phys Med Rehabil 2003;84(10):1523-1527

14 Comfort TH, Arafiles RP. Barbotage of the shoulder with imageintensified fluoroscopic control of needle placement for calcific tendinitis. Clin Orthop Relat Res 1978;(135):171-178

15 Farin PU, Jaroma H, Soimakallio S. Rotator cuff calcifications: treatment with US-guided technique. Radiology 1995;195 (3):841-843

16 Aina R, Cardinal E, Bureau NJ, Aubin B, Brassard P. Calcific shoulder tendinitis: treatment with modified US-guided fine-needle technique. Radiology 2001;221(2):455-461

17 del Cura JL, Torre I, Zabala R, Legórburu A. Sonographically guided percutaneous needle lavage in calcific tendinitis of the shoulder: short- and long-term results. AJR Am J Roentgenol 2007;189(3): W128-W134

18 Serafini G, Sconfienza LM, Lacelli F, Silvestri E, Aliprandi A, Sardanelli F. Rotator cuff calcific tendonitis: short-term and 10 -year outcomes after two-needle us-guided percutaneous treatment-nonrandomized controlled trial. Radiology 2009;252(1): $157-164$

19 Sconfienza LM, Bandirali M, Serafini G, et al. Rotator cuff calcific tendinitis: does warm saline solution improve the short-term outcome of double-needle US-guided treatment? Radiology 2012;262(2):560-566 